\title{
EP-119
}

\section{Quality of life after repair (hepatico-jejunostomy) for post-cholecysyectomy bile duct injury}

\author{
Rohit DHAWAN, Vinay KAPOOR*
}

Department of Surgical Gastroenterology, SGPGIMS, Luknow, India

Introduction: Health-related quality of life (QoL), physical component score (PCS) and mental component score (MCS), was assessed for bile duct injury sustained at cholecystectomy.

Methods: QoL was studied using Short Form-36 in 119 patients at least 24 months after repair (hepatico-jejunostomy), 50 patients who underwent uneventful cholecystectomy at least 24 months ago were controls. Effect of age, sex, type (laparoscopic, open, laparoscopic converted-to-open) of cholecystectomy, pre-repair interventions, (endoscopic and surgical) number of pre-repair interventions, Bismuth type of biliary stricture, post-repair complications, need for intervention in the follow up, total number of operations required, total duration of hospitalization on QoL was studied.

Results: Age $>50$ years $(\mathrm{n}=25)$ (PCS, MCS 44, 51 vs. 66,70 in $<30$ vs. 61,62 in $30-50 ; p<0.001,0.018)$, need for pre-repair intervention $(\mathrm{n}=102)$ (MCS 61 vs. 75; $p=0.01$ ), post-repair Clavien-Dindo III, IV complications ( $\mathrm{n}=8)$ (PCS, MCS 46, 50 vs. 64, 68; $p=0.03$, $0.04)$, need for post-repair surgical intervention ( $\mathrm{n}=28$ ) (PCS, MCS 49, 54 vs. 60, 63; $p=0.004,0.01$ ) and need for reHJ in follow up $(\mathrm{n}=16)$ (PCS 44 vs. 59; $p=0.12)$ adversely affected QoL. Patients $(\mathrm{n}=15)$ who did not require pre-repair intervention, did not have post-repair complication and did not require intervention in follow up had better QoL (PCS 63 vs. 51, MCS 82 vs. 56, $p<0.03$ ) than those $(\mathrm{n}=32)$ who required pre-repair intervention, had post-repair complication, and required intervention in follow up.

Conclusions: QoL is compromised, even after repair of BDI at biliary center; older patients, those who required pre-repair (HJ) interventions, had post-repair $(\mathrm{HJ})$ complications, required post-repair $(\mathrm{HJ})$ surgical intervention and required reHJ in follow up had poorer QoL. 